\title{
PRODUCTION DE FAMILLES DE BROCHETONS EN PETITS ÉTANGS PAR REPRODUCTION NATURELLE AMÉNAGÉE : BILAN DE TROIS ANNÉES D'EXPÉRIMENTATION ET COMPARAISON AVEC LA MÉTHOdE D'AleVINAGE
}

\author{
C. BRY (1), Y. SOUCHON (2), G. NEVEU (3), L. TREBAOL (1)
}

(1) I.N.R.A., Laboratoire de Physiologie des Poissons, 78350 JOUY-en-JOSAS

(2) Université Catholique de Lyon, Laboratoire d'Ichtyologie, 25, rue du Plat, 69288 LYON Cedex 1. Adresse actuelle : C.S.P., Délégation Régionale $n^{\circ} 5.285$, rue du 4 août, 69100 VILLEURBANNE.

(3) C.S.P., Le Paraclet, B.P. 5, 80440 BOVES

\section{INTRODUCTION}

Le "fingerling", brocheton de $50-100 \mathrm{~mm}$ pesant quelques grammes, est fréquemment retenu pour assurer un peuplement ou un repeuplement en brochets. Ce stade est en effet moins vulnérable que l'alevin à vésicule résorbée en présence de petits prédateurs et de conditions climatiques défavorables (CARBINE, 1944; FRANKLIN et SMITH, 1963; HUET, 1970). Il est par ailleurs possible d'élever en grand nombre des brochetons de quelques grammes selon différentes techniques (revues par HUET, 1976 et TIMMERMANS, 1979), alors que la production en quantité de sujets d'un été exige des surfaces considérables (HUET, 1970; TIMMERMANS, 1979).

En dépit de l'apparition récente de méthodes d'élevage intensif pour plusieurs ésocidés (RAHN, 1972; STEFFENS, 1976; GRAFF, 1978; ORME, 1978), les "fingerlings" de l'espèce Esox lucius sont très souvent produits en petits étangs, selon une formule extensive. Les avantages essentiels de l'élevage extensif sont sa simplicité et la possibilité de produire des brochetons de taille satisfaisante et de bonne qualité (TIMMERMANS, 1979). Dans ce type d'élevage, des alevins de brochet issus d'une écloserie sont introduits dans les étangs quelques jours avant l'achèvement de la résorption vésiculaire. La croissance s'effectue à partir de plancton et d'autres invertébrés, et la récolte des "fingerlings" intervient quatre à huit semaines après la mise en charge.

Les étangs à "fingerlings", généralement peu profonds et pourvus de végétation immergée, peuvent constituer un environnement approprié à la reproduction naturelle. Certains auteurs ont donc tenté de simplifier la production extensive de brochetons en introduisant dans les étangs des géniteurs (et non pas des alevins) évitant ainsi la nécessité d'une écloserie et d'opérations parfois longues et délicates, liées à la reproduction artificielle. Cette variante de l'élevage extensif a cependant conduit à des densités numériques modérées et variables : 0,4-0,8 brocheton $/ \mathrm{m}^{2}$ (Mc CARRAHER, 1957): 0.7 (BRYAN, 1967); 0,6-1,5 (FORNEY, 1968); 0,2-0,4 (ROYER, 1971); 0,08-0,8 (FAGO, 1977). $D^{\prime}$ autre part, aucune relation entre le nombre ou la taille des femelles et la production de jeunes n'a pu être mise en évidence. En fait, toutes ces expériences ayant impliqué l'introduction de plusieurs femelles par étang, l'ovulation et la fraie des différentes femelles n'ont vraisemblablement pas été synchronisées, avec pour conséquence l'apparition de familles d'alevins d'âges variés. L'hétérogénéité des tailles résultant de l'étalement des pontes a pu favoriser le développement du cannibalisme et par conséquent diminuer la densité numérique.

Nous avons donc cherché à mettre en œuvre la reproduction naturelle dans les conditions a priori les plus favorables :

1) Attribution d'une seule femelle et de deux mâles par étang, selon une mise en charge adéquate.

2) Utilisation de petits étangs peu profonds, riches en végétation et dépourvus de la plupart des prédateurs de l'alevin de brochet. 
Les performances de la reproduction naturelle ainsi aménagée ont été évaluées au cours de trois saisons consécutives et dans deux sites distincts, en utilisant des étangs de $270-400 \mathrm{~m}^{2}$. Une extension à des étangs de $1500-2000 \mathrm{~m}^{2}$ a été réalisée au cours de la seule année 1982. Dans tous les cas, la production de brochetons par reproduction naturelle aménagée a été comparée avec celle résultant de l'alevinage d'une fratrie par étang (BRY et GILLET, 1980). Une partie de ce travail a été rapportée par ailleurs (BRY et SOUCHON, 1982).

\section{MATÉRIEL ET MÉTHOdES}

Les expérimentations ont été menées dans la région de la Somme (ésociculture du Paraclet, Conseil Supérieur de la Pêche) et dans la Dombes (domaine de M. Verzier). Les petits étangs expérimentaux étaient peu profonds ( $1 \mathrm{~m}$ maximum) et complètement vidangeables (Fig. 1). La végétation des étangs était composée de roseaux Phalaris arundinacea, de Glyceria spp., de Rumex spp. et d'herbes terrestres au Paraclet, et d'Elodea canadensis dans la Dombes. Les expériences ont été réalisées en majeure partie dans des étangs de $270-400 \mathrm{~m}^{2}$ (le Paraclet et la Dombes). En 1982, des étangs plus grands (1500-2000 $\left.\mathrm{m}^{2}\right)$ ont également été utilisés au Paraclet. L'élevage extensif du brocheton par alevinage classique ou par reproduction naturelle aménagée est représenté sur la figure 2 .

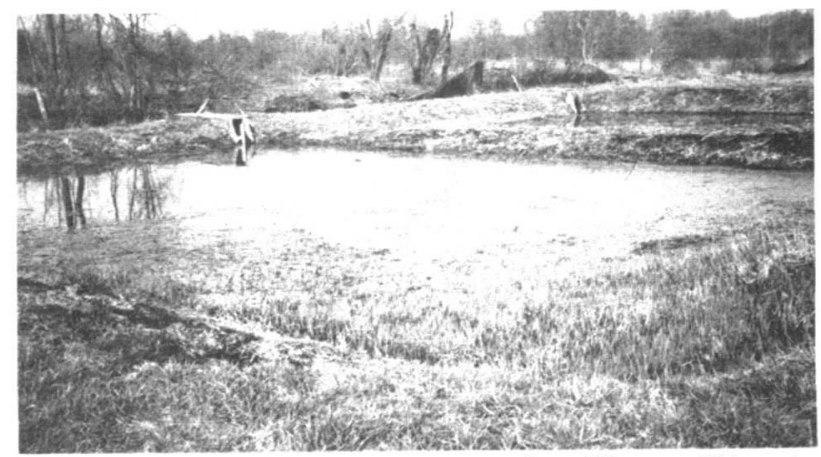

Figure 1 : Petit étang à fond enherbé utilisé pour la production de “ fingerlings" de brochet par alevinage ou par reproduction naturelle aménagée (superficie : $285 \mathrm{~m}^{2}$ ).

\section{- L'alevinage}

Aux $2 / 3$ environ de la période de résorption vésiculaire, les alevins de brochet (Esox lucius) issus de l'insémination artificielle en écloserie ont été dénombrés individuellement par siphonnage, puis déversés dans de petits étangs selon des mises en charges classiques (HUET, 1976) : 5 à 10 alevins $/ \mathrm{m}^{2}$ (tableaux 1 et 3). A chaque étang a été attribuée une fratrie, c'est-à-dire un lot d'alevins provenant des ovules d'une femelle inséminés avec le sperme de 1 à 3 mâles. L'alevinage a été effectué à des dates variant du 26 mars au 12 avril selon les années et les lots.

La mise en eau des étangs a eu lieu 10-15 jours avant l'introduction des alevins. Un dépôt modéré de fumure organique a été effectué au moment de la mise en eau au Paraclet, mais non dans la Dombes. Cinquante jours environ après le déversement, c'està-dire pendant la seconde quinzaine de mai, les étangs ont été vidangés, les brochetons dénombrés et leur poids moyen déterminé. Dans certains lots, la pesée individuelle de tous les brochetons a été réalisée à 0,1 g près, permettant une quantification précise de la variabilité pondérale. Les étangs alevinés ne renfermaient aucun poisson-fourrage.

\section{- La reproduction naturelle aménagée (R.N.A.)}

A la mi-février, c'est-à-dire 15 jours environ avant le début de la période de fraie, les étangs destinés à la R.N.A. ont été mis en eau. Quelques jours plus tard, une femelle et deux mâles ont été introduits dans chaque étang, le jour même de leur capture. Pour les lots de $270-400 \mathrm{~m}^{2}$, le poids des femelles a varié de 0,4 à 1,4 kg et celui des mâles de 0,2 a $0,8 \mathrm{~kg}$. Dans les étangs de $1500-2000 \mathrm{~m}^{2}, I^{\prime}$ 'étendue des poids a été de 2,1 à $2,8 \mathrm{~kg}$ 


\section{MILIEU NATUREL| MILIEU NATUREL SAUVAGE PROTEGE}

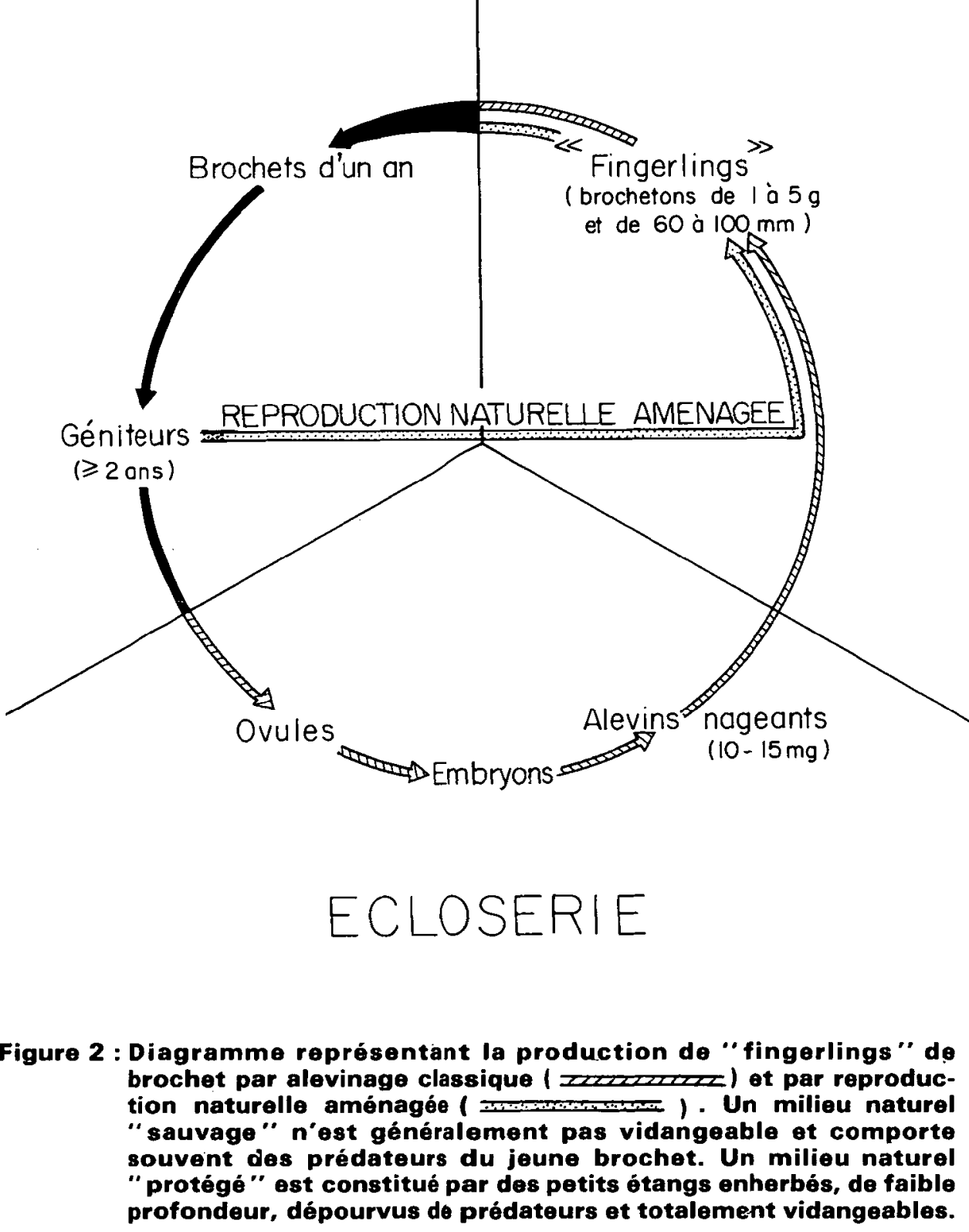


(femelles) et de 0,8 à $1,6 \mathrm{~kg}$ (mâles). En moyenne, les mises en charge ont été de $21 \mathrm{~kg} / \mathrm{ha}$ (femelles) et $25 \mathrm{~kg} / \mathrm{ha}$ (mâles) pour les étangs de $1500-2000 \mathrm{~m}^{2}$.

Compte tenu de la forte variabilité de la fécondité relative observée chez des femelles de petite taille des deux sites expérimentaux, les valeurs extrêmes approximatives de 20000 et 50000 ovocytes par kg de poids total ont été utilisées pour estimer les limites de la densité d'œufs déposés dans les étangs de $270-400 \mathrm{~m}^{2}$ (tableau 2).

En revanche, la valeur moyenne de 28000 ovocytes par $\mathrm{kg}$ de poids total a été retenue pour estimer la densité d'œufs déposés par les femelles de plus grande taille dans les étangs de $1500-2000 \mathrm{~m}^{2}$ (tableau 3). Ce choix d'une valeur moyenne de la fécondité relative s'appuie sur la variabilité modérée de ce paramètre enregistrée chez des femelles de 1,7 a $3,3 \mathrm{~kg}$ de même origine que celles utilisées en R.N.A. dans les étangs de $1500-2000 \mathrm{~m}^{2}$.

En même temps que les géniteurs brochet, du poisson-fourrage immature (brème commune Abramis brama, gardon Rutilus rutilus, rotengle Scardinius erythrophthalmus ou tanche Tinca tinca) et de taille adéquate a été introduit dans les étangs à raison généralement de $0,2 \mathrm{~kg}$ par $\mathrm{kg}$ de brochet. Un apport supplémentaire de poisson-fourrage ( $0,4 \mathrm{~kg}$ par $\mathrm{kg}$ de brochet) a été réalisé à partir de la mi-avril, après la détection de brochetons d'une vingtaine de $\mathrm{mm}$. Les étangs utilisés en R.N.A. ne renfermaient aucun poisson-fourrage consommable par les brochetons. Au Paraclet, les étangs destinés à la R.N.A. ont reçu à la mi-mars le même dépôt de fumure organique que les étangs alevinés. Une mesure hebdomadaire des températures minimum-maximum au niveau moyen de profondeur a été effectuée sur les deux sites.

Selon les lots et les années, les alevins de brochet issus de la reproduction naturelle ont pu être détectés au stade alevin en cours de résorption ou alevin nageant entre le 27 mars et le 15 avril. La recapture des géniteurs après la fraie et avant la vidange finale a été tentée en 1980, mais pas en 1981 ni 1982.

Chaque année, les étangs utilisés en R.N.A. ont été vidangés au cours de la seconde quinzaine de mai, approximativement dans l'ordre de déroulement de la reproduction. En ce qui concerne les brochetons, les mêmes mesures que dans les lots alevinés ont été effectuéés.

\section{- Comparaison de l'alevinage et de la R.N.A.}

Les dates des vidanges ont été choisies de façon à obtenir en moyenne des brochetons de même poids ( 2 à $3 \mathrm{~g}$ ) dans les deux séries d'étangs. Les données de certains lots alevinés ou de R.N.A. n'ont pu être retenues sur le plan de la production quàntitative de fingerlings en raison d'assèchements partiels survenus de façon répétée en mars-avril, de problemes de fuites importantes à la vidange ou d'une vidange trop tardive. Ces lots exclus ne figurent donc pas dans les tableaux 1, 2, 3, mais dans le cas de la R.N.A., les données de ces lots ont été prises en compte pour l'évaluation de la proportion de femelles ayant frayé et de la survie des géniteurs après reproduction naturelle.

\section{- Analyse statistique}

Les comparaisons de variances ont été effectuées à l'aide du test de Bartlett. Les moyennes (densités numériques ou biomasses) ont été comparées à l'aide du test des rangs de White. La variabilité relative des poids individuels des brochetons à l'intérieur d'un même lot a été évaluée par le cœefficient de variation (quotient de l'écart type par la moyenne en \%).

\section{RÉSULTATS}

Dans les étangs de $270-400 \mathrm{~m}^{2}$ (tableaux 1 et 2), les densités numériques moyennes de brochetons n'ont pas différé significativement entre les deux méthodes (alevinage : 1,8 brochetons $/ \mathrm{m}^{2}$ - R.N.A. : 2,3 brochetons $/ \mathrm{m}^{2}$ - $P>0,05$ ). La variabilité inter-lots du nombre de brochetons récoltés par $\mathrm{m}^{2}$ a été plus élevée dans le cas de la R.N.A. (coefficient de variation C.V. : $47 \%$; valeurs extrêmes : $0,9-4,0$ brochetons $/ \mathrm{m}^{2}$; $n=10$ ) que dans celui de l'alevinage (C.V. : $29 \%$; extrêmes : $0,9-2,6$ brochetons $/ \mathrm{m}^{2} ; n=$ 13 ) et la différence entre les variances correspondantes a été significative $(P<0,05)$. Les biomasses moyennes de brochetons obtenues par les deux méthodes n'ont pas été significativement différentes (alevinage : $54 \mathrm{~kg} / \mathrm{ha}$ - R.N.A. : $46 \mathrm{~kg} / \mathrm{ha}-\mathrm{P}>0.05$ ). Dans les conditions de mise en charge adoptées, la variabilité intra-lots du poids individuel des brochetons a été en moyenne la même dans les 2 cas (alevinage, $C . V$. $=30 \%, n=10$ R.N.A., C.V. $=30 \%, n=5$ ). 


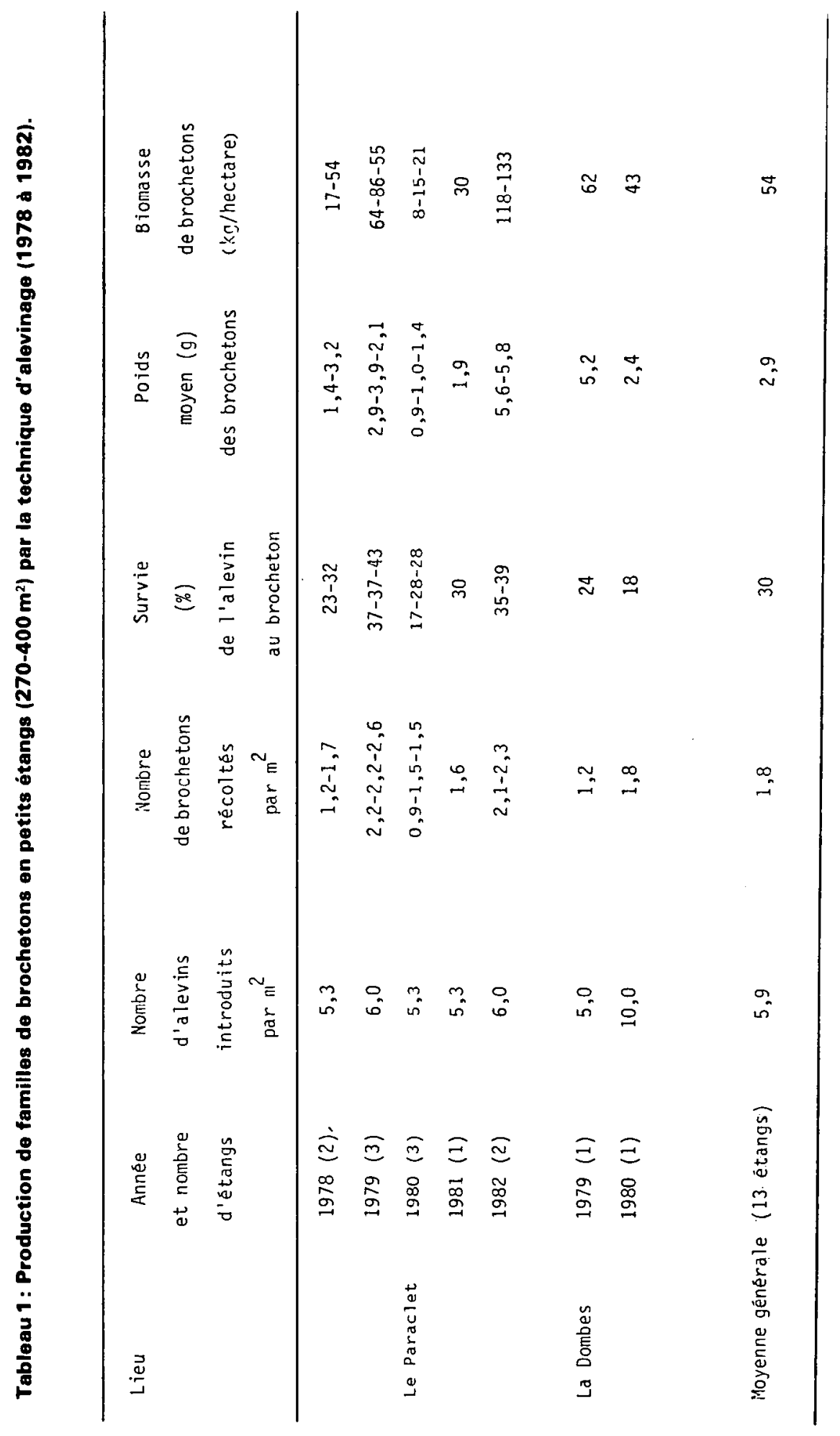




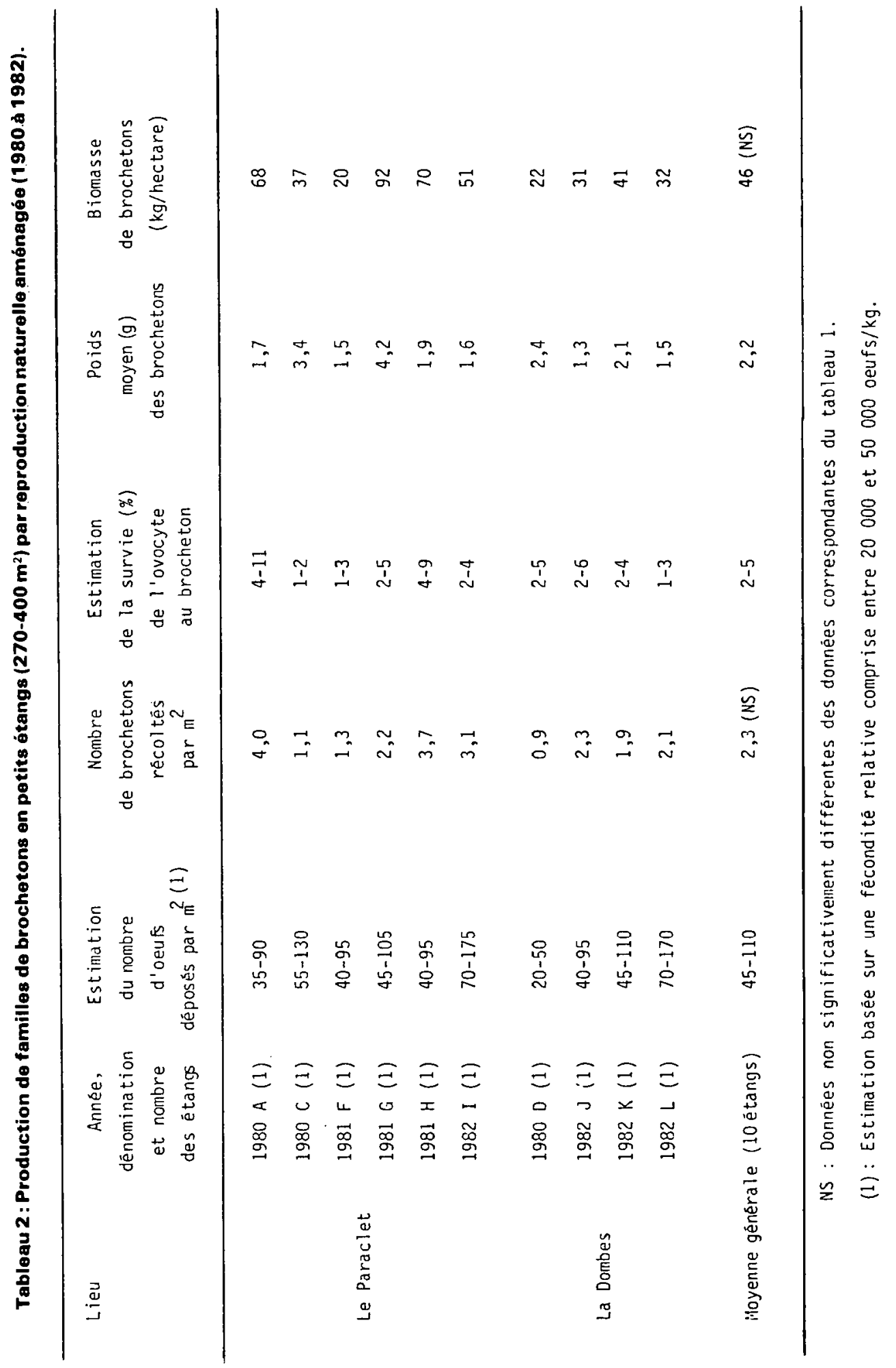




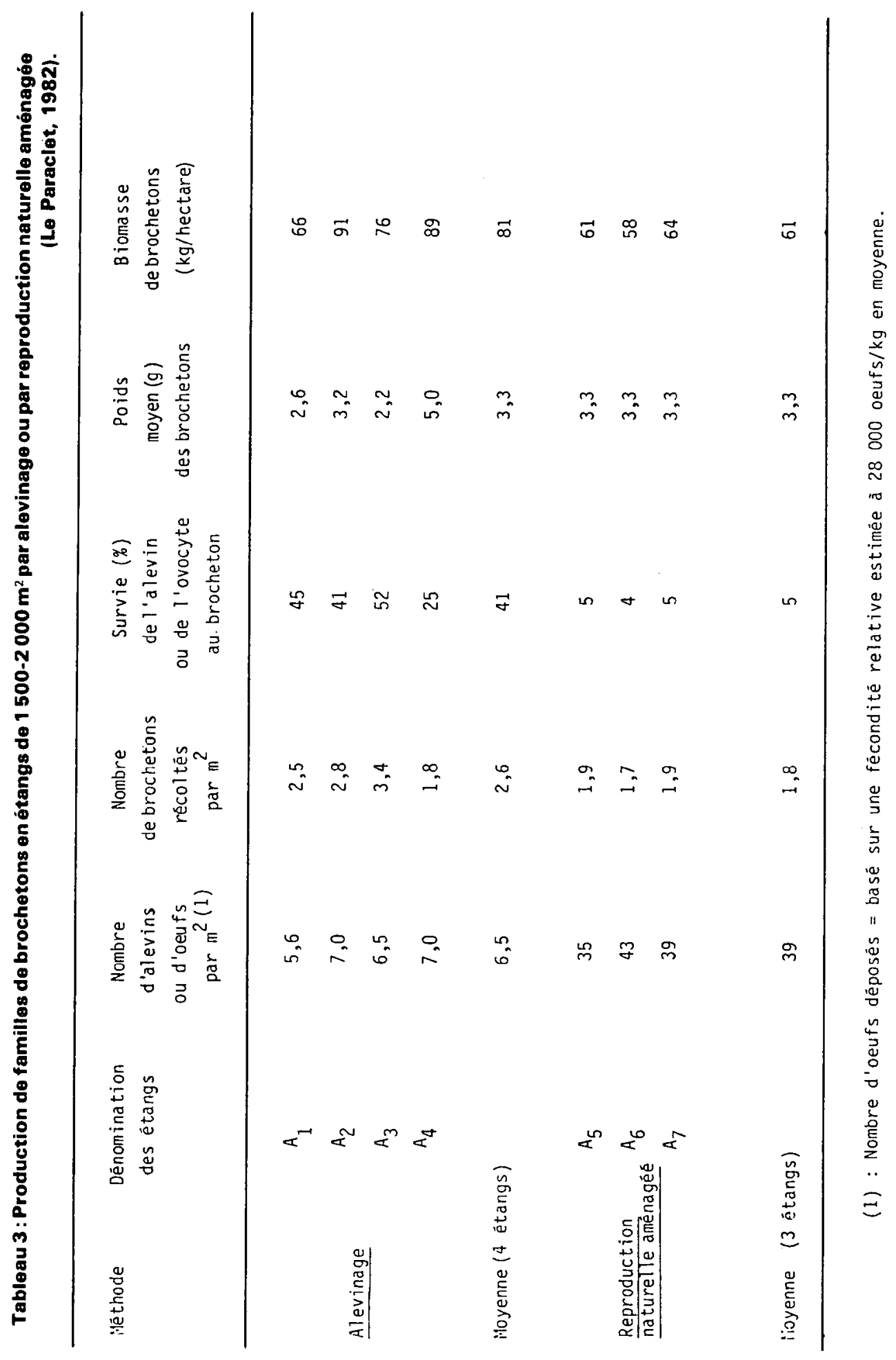


Dans les étangs de $1500-2000 \mathrm{~m}^{2}$ (tableau 3), et bien que le nombre de données ait été trop faible pour procéder à une analyse statistique, une différence sensible est apparue entre les résultats des deux méthodes : le rapport performances de la R.N.A./performances de l'alevinage, qui était de 1 pour les petits étangs, a été ici de 0,70 pour les densités numériques et de 0,75 pour les biomasses.

Pour l'ensemble des deux catégories d'étangs, le taux de survie moyen du stade alevin en fin de résorption au stade brocheton de $2-3 \mathrm{~g}$ a été de $32 \%$ par alevinage. Le taux de survie moyen du stade ovocyte au stade brocheton a été de l'ordre de $4 \%$ pour la R.N.A. Les valeurs extrêmes de la température de l'eau enregistrées entre la mi-février et la fin mai ont été $4-18^{\circ} \mathrm{C}$ au Paraclet et $4-24^{\circ} \mathrm{C}$ dans la Dombes.

Au total, 23 tentatives de reproduction naturelle aménagée ont été effectuées et 22 reproductions naturelles ont effectivement eu lieu. La seule absence de reproduction qui se soit produite résulte de la mort d'une femelle avant la fraie, peut-être liée à un nombre excessif de transferts avant son introduction en étang de reproduction. Les trois femelles utilisées en R.N.A. au Paraclet en 1980 ont été sacrifiées en avril : l'examen des ovaires a démontré que l'ovulation et l'oviposition s'étaient déroulées de façon totale dans les trois cas.

Le taux de survie global des géniteurs utilisés en R.N.A., enregistré entre la mifévrier et la fin mai (ou la mi-février et la mi-avril pour certains géniteurs en 1980) a été de $81 \%$ pour les femelles $(n=21$ ) et $62 \%$ pour les mâles $(n=42)$. Le taux de survie du poisson-fourrage introduit dans les étangs de R.N.A., mesuré à la vidange finale de fin mai, a été en moyenne de $43 \%$.

\section{DISCUSSION}

L'une des conclusions majeures de cette étude est le fait qu'une mise en charge effectuée avec des géniteurs brochet peut conduire en petits étangs à une production extensive de brochetons tout à fait comparable aux résultats obtenus par alevinage classique, à la fois en densité numérique et en biomasse. La comparaison de ces deux méthodes a été précédemment tentée par ROYER (1971): dans un plan d'eau de 2,6 ha, une production de 0,2 a 0,4 brocheton $/ \mathrm{m}^{2}$ a été obtenue à partir de géniteurs, contre moins de $0,001 / \mathrm{m}^{2}$ à partir d'alevins. Cependant, en raison d'une mortalité anormalement élevée des alevins après déversement (40\% au bout de 18 heures; près de $100 \%$ a $39-45$ jours), aucune comparaison valide n'avait pu être effectuée.

Nous n'avons pas recherché de façon systématique les effets de différentes mises en charge sur la production de brochetons. SOUCHON (1980) a précédemment démontré que si l'on alevinait des étangs de $400 \mathrm{~m}^{2}$ selon une gamme de mises en charge modérées $\left(2,25-3,75-5,0\right.$ alevins $\left./ \mathrm{m}^{2}\right)$ la densité numérique $\left(0,5-0,9-1,2 \mathrm{par} \mathrm{m}^{2}\right)$ et la biomasse $(27$ - 49 - $65 \mathrm{~kg} / \mathrm{ha})$ de brochetons étaient proportionnelles à la densité d'alevins initiale. Il est donc possible que les mises en charge adoptées pour les lots de 270-400 $\mathrm{m}^{2} \mathrm{n}$ 'aient pas été optimum. La production de brochetons dans ces petits étangs pourrait probablement être améliorée en augmentant raisonnablement les mises en charge, qu'il s'agisse d'alevinage ou de reproduction naturelle aménagée. Dans les étangs de $1500-2000 \mathrm{~m}^{2}$ en revanche, et compte tenu de la biomasse très élevée 181 $\mathrm{kg} / \mathrm{ha}$ ) obtenue par alevinage en 1982 , il est vraisemblable que la densité de 6,5 alevins $/ \mathrm{m}^{2}$ (mise en charge) était très voisine de la densité idéale permettant une production maximum dans les conditions de la saison 1982 . Les performances sensiblement inférieures de la R.N.A. dans des étangs de la même catégorie en 1982 peuvent être attribuées à une surcharge initiale. En effet, des observations répétées des grands étangs de R.N.A. ont montré l'existence dans tous les cas d'une densité d'alevins nageant extrêmement élevée, de l'ordre de 12 à 20 alevins $/ \mathrm{m}^{2}$, c'est-à-dire une densité 2 a 3 fois plus forte que dans les étangs alevinés. Cette situation surdensitaire s'est maintenue jusqu'au stade $35 \mathrm{~mm}$ environ. Par la suite, la vidange des étangs n'étant intervenue qu'au stade $85 \mathrm{~mm}$ (environ $3 \mathrm{~g}$ ). le cannibalisme s'est développé de façon considérable, entraînant une réduction massive des nombres et une forte hétérogénéité des tailles. Compte tenu de ces observations, une meilleure adaptation de la mise en charge par géniteurs à cette catégorie d'étangs reste donc à définir.

D'une façon générale, les chiffres de production cités dans ce travail figurent parmi les plus élevés qui aient été rapportés. Ceci peut être lié à la petite taille des étangs expérimentaux et à d'autres caractéristiques favorables (TIMMERMANS, 1979; BRY et GILLET, 1980). II convient par ailleurs de signaler que la très forte variabilité des 
biomasses récoltées dans les lots de $270-400 \mathrm{~m}^{2}$ (par alevinage et par R.N.A.) résulte en grande partie du fait que les brochetons ont été récoltés à des tailles très variables, leur poids moyen s'étendant de 0,9 à $5,8 \mathrm{~g}$ selon l'époque de la vidange.

Dans des conditions d'environnement identiques, le nombre de brochetons récoltés par $\mathrm{m}^{2}$ dans les petits étangs s'est révélé plus variable dans le cas de la R.N.A. que dans celui de l'alevinage. Ce point peut s'expliquer par (1) la variabilité de la fécondité relative des petites femelles utilisées et par (2) la variabilité d'une ponte à l'autre des taux de survie précoce, peut-être liée à des différences de qualité des gamètes selon les géniteurs et au fait que la fraie s'est déroulée à des époques différentes (entre fin février et fin mars) selon les lots. La variabilité inter-lots de la densité numérique de brochetons demande en fait à être étudiée plus avant puisque dans les étangs de 1500-2000 m², c'est au contraire dans les lots alevinés que ce paramètre a été le plus élevé.

Le taux de survie moyen du stade ovocyte au stade brocheton de $2-3 \mathrm{~g}$ est de l'ordre de $4 \%$ par R.N.A., ce qui correspond à une production d'environ 1200 brochetons par $\mathrm{kg}$ de femelle. Ce taux de survie est 10 à 50 fois plus élevé qu'en milieu naturel "sauvage" (CARBINE, 1944; FRANKLIN et SMITH, 1963), mais inférieur d'un facteur 1,3 a 5 aux taux obtenus par reproduction artificielle et alevinage (BRY, données non publiées). Cette dernière différence pourrait cependant être en partie compensée par la survie élevée des géniteurs après R.N.A., compte tenu des mortalités observées après les manipulations liées aux opérations de reproduction artificielle (BRY, MAISSE, NEVEU, données non publiées).

En conclusion, il convient d'insister sur le fait que des productions élevées de brochetons ont été obtenues par reproduction naturelle aménagée pendant trois années et ce, malgré des périodes répétées de basses températures de l'eau $\left(4-5^{\circ} \mathrm{C}\right)$ au cours des mois de mars dans les deux sites expérimentaux. Dans les conditions où nous l'avons pratiquée, la R.N.A. s'est avérée être une technique reproductible, ne demandant qu'une main-d'couvre réduite et peu de technicité, et permettant de faire l'économie d'une écloserie. La recapture des géniteurs peu après la fraie ne s'impose pas si du poissonfourrage leur a été fourni de façon appropriée. Enfin, la survie élevée des géniteurs après reproduction permet d'envisager leur utilisation pendant plusieurs années consécutives.

\section{REMERCIEMENTS}

Cette étude a été prise en charge par le Conseil Supérieur de la Pêche. Nos remerciements vont à Messieurs.J.P. CAVITTE et J. VERZIER pour l'accueil qu'ils nous ont réservé ainsi qu'à G. BURGER, R. DEMANET, S. FAUCHILLE, J.P. HISEUX, R. PUYPE, S. QUINTON et $\mathbf{G}$. SLOF pour leur collaboration.

\section{RÉfÉRENCES}

BRY C. and C. GILLET, 1980. Reduction of cannibalism in pike (Esox /ucius) fry by isolation of full-sib families. Reproduction Nutrition Développement, 20, 173-182.

BRY C. and Y. SOUCHON, 1982. Production of young northern pike families in small ponds : natural spawning versusfry stocking. Transactions of the American Fisheries Society, 111, 476-480.

BRYAN J.E., 1967. Northern pike production in Phalen Pond, Minnesota. Journal of the Minnesota Academy of Science, 34 (2), 101-109.

CARBINE W.F., 1944. Egg production of the northern pike, Esox /ucius L., and the percentage of survival of eggs and young on the spawning grounds. Papers of the Michigan Academy of Science, 29, 123-137.

FAGO D.M., 1977. Northern pike production in managed spawning and rearing marshes. Wisconsin Department of Natural Resources. Technical Bu/letin 96.

FORNEY J.L., 1968. Production of young northern pike in a regulated marsh. New York Fish and Game Journal, 15 (2). 143-154.

FRANKLIN D.R. and SMITH L.L., Jr. 1963. Early life history of the northern pike with special reference to the factors influencing the numerical strength of year classes. Transactions of the American Fisheries Society, 92, 91-110. 
GRAFF D.R., 1978. Intensive culture of esocids: the current state of the art. American Fisheries Society Special Publication, 11, 195-201.

HUET M., 1970. Traité de pisciculture. Editions Ch. de Wyngaert, Bruxelles, Belgique.

HUET M., 1976. Reproduction, incubation et alevinage du brochet. EIFAC (European Inland Fisheries Advisory Committee) Technical Paper 25, 147-163.

Mc CARRAHER D.B., 1957. The natural propagation of northern pike in small drainable ponds. The Progressive Fish-Cu/turist, 19. 185-187.

ORME L.E., 1978. The status of coolwater fish diets. American Fisheries Society Special Publication. 11, 167-171.

RAHN J., 1972. Hv Produktion in Intensivanlagen. Zeitschrift für die Binnenfischerei der DDR. 19, 124-127.

ROYER L.M., 1971. Comparative production of pike fingerlings from adult spawners and from fry planted in a controlled spawning marsh. The Progressive Fish-Culturist. 33, 153-155.

SOUCHON Y., 1980. Effet de la densité initiale de peuplement sur la survie et la croissance du brochet (Esox lucius L.) élevé jusqu'au stade de brocheton (45 jours). Pages 309-316 in R. Billard, éditeur. La Pisciculture en Etang. Institut National de la Recherche Agronomique Publications, Paris, France.

STEFFENS $\dot{W}$., 1976. Hechtzucht. Zeitschrift für die Binnenfischerei der DDR, 23, 360371.

TIMMERMANS J.A., 1979. Culture of fry and fingerlings of pike. Esox lucius. EIFAC (European Inland Fisheries Advisory Committee) Technical Paper, 35, supplement 1, 177-183.

\section{RÉSUMÉ}

La méthode de Reproduction Naturelle Aménagée (R.N.A.) consiste à introduire à la mi-février et selon une mise en charge adéquate une femelle brochet et deux mâles dans un milieu naturel " protégé" (petit étang enherbé et peu profond, doté de poissonfourrage, dépourvu de prédateurs et dont le niveau d'eau est stable). La récolte des brochetons issus de la fraie et la récupération des géniteurs s'effectuent simultanément par vidange à la fin mai. La R.N.A. a été mise en'œuvre en 1980-81-82 dans des batteries de petits étangs (270-400 $\mathrm{m}^{2}$ ) situées au Paraclet (Somme) et dans La Dombes. Les performances de la R.N.A. ont été comparées aux résultats obtenus dans les mêmes milieux par alevinage classique (introduction d'une fratrie d'alevins en fin de résorption par étang) :

1. Les productions numériques moyennes de brochetons de $2-3 \mathrm{~g}$ ne diffèrent pas significativement entre les deux méthodes (Alevinage : 1,8 brochetons $/ \mathrm{m}^{2}-$ R.N.A. : 2,3 brochetons $\left./ \mathrm{m}^{2}\right)$.

2. La variabilité inter-lots du nombre de brochetons par $\mathrm{m}^{2}$ est significativement plus élevée dans le cas de la R.N.A. (coefficient de variation c.v. : $47 \%$; valeurs extrêmes : $0,9-4,0$ brochetons $/ \mathrm{m}^{2}$ ) que dans celui de l'alevinage (c.v. : $29 \%$; extrêmes : 0,9-2,6 brochetons $\left(\mathrm{m}^{2}\right)$.

3. Les biomasses moyennes de brochetons ne sont pas significativement différentes (Alevinage : $54 \mathrm{~kg} / \mathrm{ha}$ - R.N.A. : $46 \mathrm{~kg} / \mathrm{ha}$ ).

4. Dans les conditions de mise en charge adoptées, la variabilité intra-lots du poids individuel des brochetons est en moyenne la même pour les deux méthodes (c.v. moyen : $30 \%$ ).

5. Le taux de survie moyen du stade ovocyte au stade brocheton de $2-3 \mathrm{~g}$ est de l'ordre de $4 \%$ par R.N.A., ce qui correspond à une production d'environ 1200 brochetons par $\mathrm{kg}$ de femelle. Ce taux de survie est élevé pour une reproduction naturelle, mais inférieur d'un facteur 1,3 à 5 aux taux obtenus par reproduction artificielle et alevinage. Cette différence pourrait cependant être en partie compensée par la survie élevée des géniteurs après R.N.A. : 81 \% pour les femelles, $62 \%$ pour les mâles.

Une tentative d'extension de la Reproduction Naturelle Aménagée à des étangs de $1500-2000 \mathrm{~m}^{2}$ a été conduite au Paraclet en 1982 : la production moyenne (1,8 brochetons $/ \mathrm{m}^{2} ; 61 \mathrm{~kg} / \mathrm{ha}$ ) a été sensiblement inférieure à celle obtenue dans des 
étangs-témoins alevinés $\left(2,6\right.$ brochetons $\left./ \mathrm{m}^{2} ; 81 \mathrm{~kg} / \mathrm{ha}\right)$. La différence observée résulte très vraisemblablement d'une surcharge initiale dans les lots de R.N.A. Une meilleure adaptation de la mise en charge par géniteurs à cette catégorie d'étangs reste donc à définir.

Ces différents résultats suggèrent que, dans de petits étangs enherbés propices à la fraie et sous réserve d'une mise en charge adéquate, la méthode de Reproduction Naturelle Aménagée peut constituer une variante de l'élevage extensif du brocheton aussi productive que l'alevinage classique. Dans les conditions où nous l'avons pratiquée, la R.N.A. s'est avérée être une technique reproductible, ne demandant qu'une main-d'œuvre réduite et peu de technicité, et permettant de faire l'économie d'une écloserie.

De plus, la survie élevée des géniteurs après la fraie permet d'envisager leur utilisation pendant plusieurs années consécutives.

\section{SUMMARY}

Production of young northern pike families in small ponds from managed natural spawning: results from a three year experiment and comparison with the fry stocking method.

The method of managed natural spawning (M.N.S., or R.N.A. in the French text) implies the stocking in mid-february of one northern pike female and two males, according to an adequate biomass density, in a natural and "protected" environment (i.e. small, grassy and shallow ponds, containing forage-fish but no predator, and with a constant water level). The northern pike juveniles produced from natural spawning are harvested together with the spawners by draining the ponds at the end of may. The M.N.S. technique was experimented in 1980-81-82 in small ponds $\left(270-400 \mathrm{~m}^{2}\right)$ located at le Paraclet (Somme) and in La Dombes. The productions from M.N.S. were compared with the results obtained when families of hatchery-reared fry are stocked into ponds :

1. The average numerical densities of young northern pike (mean weight : $2-3 \mathrm{~g}$ ) from the two methods were not significantly different (Fry stocking : 1.8 young $/ \mathrm{m}^{2}-$ M.N.S. : 2.3 young $/ \mathrm{m}^{2}$ ).

2. The inter-pond variability of the numerical density was significantly higher in the lots stocked with spawners (coefficient of variation c.v. : $47 \% ;$ range $: 0.9-4.0$ young $/ \mathrm{m}^{2}$ ) than in the ponds stocked with fry (c.v. : $29 \%$; range : 0.9-2.6 young $/ \mathrm{m}^{2}$ ).

3. The two methods provided comparable biomasses of juveniles (Fry stocking : 54 $\mathrm{kg} / \mathrm{ha}$ - M.N.S. : $46 \mathrm{~kg} / \mathrm{ha}$ ).

4. With the stocking rates that were used, the average intra-pond variability of young northern pike individual weight was the same for both methods (c.v. : $30 \%$ ).

5. The average egg to juvenile survival rate was about $4 \%$ in the case of M.N.S. Such a rate is high for natural spawning, but lower by 1.3 to 5 -fold than the survival rate following artificial reproduction and fry stocking. However, this difference could be partly offset by the high survival rate of spawners after M.N.S. : $81 \%$ for the females, $62 \%$ for the males.

The M.N.S. technique was also experimented in larger ponds $\left(1500-2000 \mathrm{~m}^{2}\right)$ in 1982 at Le Paraclet. The average production from M.N.S. (1.8. young $/ \mathrm{m}^{2} ; 61 \mathrm{~kg} / \mathrm{ha}$ ) was lower than in control ponds stocked with fry $\left(2.6\right.$ young $\left./ \mathrm{m}^{2} ; 81 \mathrm{~kg} / \mathrm{ha}\right)$. Such a difference probably results from overstocking in the M.N.S. ponds. The stocking density of female spawners (in terms of biomass) should therefore be better adjusted to that category of ponds.

Our results suggest that, if the stocking rate is adequate, the method of managed natural spawning in small grassy ponds may be an extensive culture technique as productive as the more traditional fry-stocking procedure. In our working conditions, the M.N.S. technique proved dependable, requiring minimal manpower and little technical skill, and sparing hatchery costs. In addition, the high survival rate of post-spawning adults makes their use possible for several years in succession. 